\title{
MATHEMATICAL MODELING OF FEEDBACK CONTROL OF GRAVITATIONAL WAVES
}

\author{
ANKUSH RAI*, JAGADEESH KANNAN R
}

School of Computing Science \& Engineering, VIT University, Chennai, Tamil Nadu, India. Email: ankushresscie@gmail.com

Received: 28 December 2016, Revised and Accepted: 10 May 2017

\begin{abstract}
We present an analysis for gravitation through space mass quantization technique where gravitational waves are the fundamental interaction through the distinct state of quantization of mass and space while remaining mathematically compatible with general relativity. It also encompasses issues such as the notion of space-radiative expansion leading to order in the large radius expansion, zero-point energy and also space contraction is introduced that sets the physical scales and subleading terms. We discuss conditions under which gravitational waves are transformed locally under space mass action. A proposal for improving the current use of scientific methods and mechanism to forge a model of gravitational waves is given. We refer this physical interpretation in rest of the text as "Quantize Field Theory."
\end{abstract}

Keyword: Gravitation, Formulation-theory and mathematical, Experimentation.

(C) 2017 The Authors. Published by Innovare Academic Sciences Pvt Ltd. This is an open access article under the CC BY license (http://creativecommons. org/licenses/by/4. 0/) DOI: http://dx.doi.org/10.22159/ajpcr.2017.v10s1.19980

\section{INTRODUCTION}

It took over one century to come up with reasoning the origin of gravitational waves after Einstein's general relativity (GR). It has provided primarily mathematical answers to the question of how gravitational waves works but had little to say about the "why" $[1,2]$. When pressed for the physics involved in gravitation, relativists these days are partial to the "geometric interpretation" of GR because it comprehends the reader that how bodies accelerate in a gravitational field but it devoid the reader's comprehension from the cause of such basic phenomenon [3-5]. Such unanswered and left out half explanation of gravitational waves derelict the truth and thus escort reader in the vortex of several assumptions. I here bestow derivations of proximate of gravitational waves origin and edify Einstein's GR.

Gravitational waves at large distances pose some of the most difficult puzzles in contemporary gravitational physics. The nature of dark matter is the most prominent one, at a somewhat larger scale, both in terms of actual size and in terms of scientific credibility; there is zero-point energy that imbalance our theoretical manifestation of cosmology [6-8]. The solution of this unexplained phenomenon could deepen our understanding of the universe and also provide us tools for more scientific exploration rather than limiting ourselves from the whirlpool of speculation and uncertainties, we'll have something absolute in our hands to mold and engineer gravitational waves. The dogma presented in the following pages conceivably constitutes the lacking percept $[9,10]$. Now, we'll proceed the "Quantize Field Theory" to demonstrate the evolving gravitational waves through a different state of space mass quantization and its consequence causing expansion of the universe and formulation of Casimir force [11]. The simulation of the equations represented below is achieved using the methods mentioned in Rai et al. [12-16].

\section{QUANTIZE FIELD THEORY}

In classical mechanics as well as in special theory of relativity, the mass at coordinates in space has a direct physical meaning. To say that, a mass at a point $\mathrm{P}$ in a space means the projection of the event by $\mathrm{m}$ at $\mathrm{P}$ on the axis of the dimensions. Furthermore, there is a standard clock time $t$ associated with the event at $\mathrm{P}$ where $\mathrm{m}$ is the cause of the event.

It is necessary for the reader's reflection of underlying concept to be independent of sleight to connect him with the mathematical definition of space. This is readily understood if we envisage the whole process of quantizing space where gravitational waves are quantized from the generalization of the action function for a particle. The action for a free particle is given by:

$$
S=-\alpha \int_{\alpha}^{0} d s
$$

Where $\alpha$ is the quantity which characterizes the particle. Applying relativistic mechanics, the action can be written in the form:

$$
S=\int_{t 1}^{t 2} L d t=-\int_{t 1}^{t 2} \alpha c \sqrt{1-\left(\frac{v}{c}\right)^{2}} d t
$$

Where, $L$ is the Lagrange's function and represented as $L=-\alpha c \sqrt{1-\left(\frac{v}{c}\right)^{2}}$. From the remnant of preconceived notion of classical mechanics the determined Lagrange's function for a free particle is $L=a v^{2} . v$ is the speed of the particle, and a is a hypothetical quantity which is given as: $a=m / 2$. Here $m$ is the mass of the particle [17]. The results of our measuring of $m$ are verifications of the indistinguishable about the kind of mass (whether it be gravitational mass $m_{g}$ or inertial mass $m_{i}$ ).

If the limit $c \rightarrow \infty$ the correlation between a and $a$ can be expressed below. Where, relativistic expression $L$ is reduced to the classical expression.

$L=a v^{2}$ (classical expression)

$L=a^{V^{2}} / 2 c$ (relativistic expression)

Now, if $\alpha=2 a c=m c$, we get

$L=a v^{2}$. Following the first axiom is given, we will have $m=m_{g}$. Therefore, $a=m_{g} / 2$.

The generalized expression for the action for a free particle is of the form:

$\delta=-m g c \int_{a}^{b} d s$ 
Or

$$
\delta=\int_{t 1}^{t 2} m_{g} c^{2} \sqrt{1-\left(\frac{v}{c}\right)^{2} d t}
$$

But the integral of $\delta=\int_{t 1}^{t 2} m_{g} c^{2} \sqrt{1-\left(\frac{v}{c}\right)^{2} d t}$ is preceded by the plus sign as it cannot have a minimum. Thus, the integrand of Equation (2) must always be positive. Therefore, if $m_{g}>0$ then $t>0$ or if $m_{g}<0$ then $t<0$.

We know that the momentum $\vec{p}=\frac{\overrightarrow{\partial L}}{\partial r}$ and $L=-m_{g} c^{2} \sqrt{1-\left(\frac{v}{c}\right)^{2}}$

$$
\vec{p}=\frac{m_{g}}{\sqrt{1-\left(\frac{v}{c}\right)^{2}}}=M_{g} \cdot \vec{v}
$$

The inertial force $F_{i}=\overrightarrow{d p} / d t$ which acts on the particle in two ways:

1. $F_{i}$ is perpendicular to speed.

$$
F_{i}=\frac{m_{g}}{\sqrt{1-\left(\frac{v}{c}\right)^{2}}} \frac{\overrightarrow{d v}}{d t}
$$

2. $\quad F_{i}$ is in the direction of speed.

$$
F_{i}=\frac{m_{g}}{\left\{\sqrt{1-\left(\frac{v}{c}\right)^{2}}\right\}^{\frac{3}{2}}} \frac{\overrightarrow{d v}}{d t}
$$

The energy of the particle is given by:

$$
E_{g}=\vec{p} \vec{v}-L=\frac{m_{g} c}{\sqrt{1-\left(\frac{v}{c}\right)^{2}}} \text { or } E_{g}=M_{g} c^{2}
$$

The introduction of $E_{g}$ serves no other purpose than to facilitate the description of the totality.

At rest, the particle's gravitational potential energy is $E_{g o}=m_{g} c^{2}$

Equation (7) can be represented in the manipulated form as:

$$
E_{g}=m_{g} c^{2}-\frac{m_{g} c_{i}}{\sqrt{1 g\left(\frac{v}{c}\right)^{2}}}-m_{g} c_{m}
$$

$$
=\frac{m_{g}}{m_{i}}\left[m_{i} c^{2}+\left[\frac{m_{i} c^{2}}{\sqrt{1 i\left(\frac{v}{c}\right)^{2}}}-m_{i} c^{2}\right]\right]=\frac{m_{g}}{m_{i}}\left(E_{i o}+E_{k i}\right)=\frac{m_{g}}{m_{i}} E_{i}
$$

In analogy with the gravitational energy at rest the inertial energy at rest $E_{i o}=m_{i} c^{2}$. Thus, the total inertial energy is $E_{i}=E_{i o}+E_{k i}\left(E_{k i}=\right.$ Kinetic inertial energy).

From Equation (6) and (8),

$$
E_{i}=\frac{m_{i} c}{\sqrt{1 i\left(\frac{v}{c}\right)^{2}}}=M_{i} c^{2}
$$

For $v<<c, E_{i}=m_{i} c^{2}+1 / 2 m_{i} v^{2}$

Comparing Equation (6) and (8)

$E_{k g}=\frac{m_{g}}{m_{i}} E_{k i}$

Gravitational field produced by a particle of gravitational mass $m_{g}$ depends on the particle's gravitational energy $E_{g}$. For this, we can write as:

$g=-\frac{G E_{g}}{r^{2} c^{2}}=-\frac{G M_{g} c^{2}}{r^{2} c^{2}}=\frac{G M_{g}}{r^{2}}$

Where, $M_{g}$ is the relativistic gravitational mass.

Since it is well known that gravitational force is conservative. Thus, the argument of the above condition can be expressed as:

$\Delta E_{g}=-\Delta E_{i}$

Thus,

$E_{i}=E_{i o}+E_{i}$ and $E_{g}=E_{g o}+\Delta E_{g}=E_{g o}-\Delta E_{i}$

$E_{g}+E_{i}=E_{g o}+E_{i o}$

Comparing between Equations (6) and (8) reveals that $E_{g o}=E_{i o}$ as a consequence of which;

$E_{g}+E_{i}=E_{g o}+E_{i o}=2 E_{i o}$

However, $E_{i}=E_{i 0}+E_{k i}$ Therefore, Equation (16) can be written as:

$E_{g}=E_{i o}-E_{k i}$

Substituting $E_{i o}=E_{i}-E_{k i}$ gives:

$E_{i}-E_{g}=2 E_{k i}$

The correlation between gravitational energy and momentum can be derived by squaring and comparing Equations (3) and (6).

$\frac{E_{g}^{2}}{c^{2}}=p^{2}+m_{g}^{2}$

The Hamiltonian function can be expressed as:

$H=c \sqrt{p^{2}+m_{g}^{2} c^{2}}$

We shall find hereafter the expression for the energy of a particle in periodic motion inside a cubical box of edge length $L$.

i.e. $E_{n}=\frac{n^{2} h^{2}}{8 m_{g} L^{2}} n=1,2,3$

The maximum edge length of a cubical box whose maximum diameter $d_{\max }$ is given as:

$d_{\text {max }}=L_{\text {max }} \sqrt{3}$ 
$d_{\max }$ is equal to the maximum diameter of the universe. The minimum energy of a particle at rest $m_{g} c^{2}=m_{i} c^{2}$.

Therefore,

$$
\frac{n^{2} h^{2}}{8 m_{g} L^{2}}=m_{g} c^{2}
$$

Or

$$
m_{g}=\frac{n h}{c L_{\max } \sqrt{8}}
$$

From consideration of relativistic mass $M_{g^{\prime}}$ it follows that,

$M_{g}(\min )=m_{g}(\min )$

From the box normalization, we conclude that the propagation number $|\vec{K}|=\frac{2 \pi}{\lambda}$ is restricted to the values $|\vec{K}|=n \frac{2 \pi}{L}$. Thus, we find $L=n \lambda$, and now we retrieve that $n_{\max }=\frac{L_{\max }}{\lambda_{\min }}$ and $L_{\min }=n_{\min } \lambda_{\min }=\lambda_{\min }$. Hence,

$L_{\max }=n_{\max } \lambda_{\max }$

Also,

$L_{\max }=n_{\max } L_{\min }$

Finally,

$L=n L_{\min }$

It can also be represented of the form as:

$\mathrm{L}=L_{\max } / n$

Multiplying Equations (26) and (27) by $\sqrt{3}$ and reminding that $d=L \sqrt{3}$, we obtain,

$d=n d_{\min }$ or $d=\frac{d_{\max }}{n}$

Having seen in the forgoing formulation, we comprehend that at the point to proclaim that space is quantized.

$M_{g(\max )}=\frac{n_{\max } h}{c L_{\min } \sqrt{8}}$

From Equation (25),

$M_{g(\max )}=\frac{n_{\max }^{2} h}{c L_{\max } \sqrt{8}}$

Now, comparing Equations (30) and (22) reveal that,

$M_{g(\max )}=n_{\max }{ }^{2} m_{g(\min )}$

$\mathrm{Or}$

$M_{g}=n^{2} m_{g(\min )}$

Equation (31) shows that gravitational mass is quantized. Substitution of Equation (32) into (12) gives:
$g=-\frac{G M_{g}}{r}=n^{2}\left(-\frac{G m_{g(\min )}}{\left(r_{\max } / n\right)^{2}}\right)=n^{4} g_{\text {min }}$

We may now look on objective to distinguish gravitational Hamiltonian $H_{g}$ from inertial Hamiltonian $H_{i}$

$$
H_{i}=c \sqrt{p^{2}-m_{i}^{2} c^{2}}
$$

As a direct consequence of it Equation (17) can be written as:

$H_{i}+H_{g}=\Delta H_{i}$

The momentum variation yields $\Delta H_{i}$ variation.

$$
\Delta H_{i}=\sqrt{(p+\Delta p)^{2} c^{2}-m_{i}^{2} c^{4}} \sqrt{p^{2} c^{2}-m_{i}^{2} c^{4}}
$$

Substituting Equations (19), (34), (36) into (35) and putting $p=0$.

$$
m_{g}^{2} c^{2}+m_{i}^{2} c^{2}=2\left(\sqrt{\Delta p^{2} c^{2}-m_{i}^{2} c^{4}}-m_{i}^{2} c^{2}\right)
$$

Thus, the generalized equation between gravitational and inertial mass is given as:

$m_{g}=m_{i}-2\left[\sqrt{12\left(\frac{\Delta p}{m_{i} c}\right)^{2}}-1\right] m_{i}$

Also, for $\Delta p>m_{i} \mathrm{c}(\sqrt{5} / 2) ; m_{g}$ will be negative.

Therefore, Equation (37) can be written in the form:

$$
(p+\Delta p)=\frac{\left(m_{g}-\Delta m_{g}\right)(v+\Delta v)}{\sqrt{1-(v+\Delta v)^{2} / c^{2}}}
$$

For $v=0, p=0$. Therefore, above equation reduced to:

$$
\Delta p=\frac{\left(m_{g}-\Delta m_{g}\right) \Delta v}{\sqrt{1-\Delta v^{2} / c^{2}}}
$$

From Equation (15) we obtain,

$E_{g}=2 E_{i o}-E_{i}=2 E_{i o}-\left(E_{i o}+\Delta E_{i}\right)=E_{i o}+\Delta E_{i}$

But from Equation (13) $-\Delta E_{i}=\Delta E_{g}$

Therefore, $E_{g}=E_{i o}+\Delta E_{g}$ or $m_{g}=m_{i}+r m_{g}$. Replacing $\mathrm{m}_{\mathrm{g}}+\mathrm{Rm}$ by $m_{i}$ in above Equation, we obtain,

$$
\Delta p=m_{i} \frac{\Delta v}{\sqrt{1-\Delta v^{2} / c^{2}}} \text { or } \frac{\Delta p}{m_{i} c}=\frac{v / c}{\sqrt{1-v^{2} / c^{2}}}
$$

Substituting the value so obtained in Equation (37), 
$m_{g}=m_{i}-2\left[\left(12^{2} / c^{2}\right)^{-\frac{1}{2}}-1\right] m_{i}$

Put $v=0$, we get,

$m_{g}=m_{i}$

It follows that the same as been done previously that $m_{g}=n^{2} m_{g}(\mathrm{~min})$ becomes $m_{i}=n^{2} m_{i}(\min )$

$F_{i}=m_{i} \vec{a}$ is the inertial force and $F_{g}=m_{g} \vec{g}$ is the gravitational forces. We already had proven $m_{g}=m_{i}$. From Equation (5) and (12),

$$
\begin{aligned}
& \frac{m_{g}}{\left(1-\left(\frac{v}{c}\right)^{2}\right)^{\frac{3}{2}}} \vec{a}=G \frac{m_{g}^{\prime}}{r^{\prime}\left(\sqrt{1-\left(\frac{v}{c}\right)^{2}}\right)^{2}} \frac{m_{g}}{\sqrt{1-\left(\frac{v}{c}\right)^{2}}} \\
& =\left[\frac{G m_{g}^{\prime}}{r^{\prime 2}}\right]_{\left(1-\left(\frac{v}{c}\right)^{2}\right)^{\frac{3}{2}}}=\vec{g} \frac{m_{g}}{\left(1-\left(\frac{v}{c}\right)^{2}\right)^{\frac{3}{2}}}
\end{aligned}
$$

$\vec{a}=\vec{g}$

Thus, the rule of equivalence is preserved.

In Einstein's theory of special relativity, the dynamics of the free particle in constricted space is described as:

$\delta S=0$

From Equation (1) we have,

$\delta S=-m_{g} c \delta \int d s=0$

Let $S_{g}$ and $S_{m}$ be the action of the constricted field and the action of matter.

Therefore,

$\delta\left(S_{g}+S_{m}\right)=0$

$\delta S_{g}=\frac{c^{3}}{16 \pi G} \int\left(R_{i k}-\frac{1}{2} g_{i k} R\right) \delta g^{i k} \sqrt{-g} d \Omega$

$\delta S_{m}=\Omega \frac{1}{2 c} \int T_{i k} \delta g^{i k} \sqrt{-g} d \Omega$

Where,

$$
\begin{aligned}
& T_{i k}=\left(P+\in_{g}\right) \mu_{i} \mu_{k}+P g_{i k} \text { and } \in_{g}=\rho_{g} c^{2} \\
& \frac{c^{3}}{16 \pi G} \int\left(R_{i k}-\frac{1}{2} g_{i k} R-\frac{8 \pi G}{c^{4}} T_{i k}\right) \delta g^{i k} \sqrt{-g} d \Omega=0 \\
& R_{i k}-\frac{1}{2} g_{i k} R-\frac{8 \pi G}{c^{4}} T_{i k}=0
\end{aligned}
$$

From the resultant equations of space mass quantization, our intuition felt a sense of realization to basic physical laws. These can be summed as "Quantize Field Theory:"
Inferring the idea from difference of state of quantization (Equations 25 and 32) of mass and space that mass quantize at the square of integers than that by space. Thus, we state this as "Space is a single state motif which represents mutiny and tends to devitalize the volume quantized by matter." The derivation of GR's equation (Equation 44) from the action of matter in space follows "Space is not an entity inflicting dimensions only, but it's a mold which pilot matter to manifest its physical properties when embodied in it."

Axiom: 1. "Space imitates symmetry due to quantization." That is, space preserves the particle state. Its verification is given below:

In the following we consider equilibrium condition and field equations of space filled with matter under its constriction and the opposing force of electric repulsion due to the disparity of charge distribution as the matter suffers contraction that eventually is of the form of gravitational force derived from distinct space mass quantization in Equation (44). Choosing comoving coordinates $(r, \theta, \varphi)$ the most general line element exhibiting spherical symmetry can be put in the form:

$d s^{2}=e^{\alpha} d t^{2}-e^{\beta}\left[e^{\lambda} d r^{2}+r^{2} d \Omega^{2}\right]$

Where,

$d \Omega^{2}=d \theta^{2}+\sin \theta^{2} \theta d^{2}$

The field equations $R_{i k}-\frac{1}{2} \delta_{i k} R=-8 \pi T_{i}^{k}$ take the form:

$$
\begin{aligned}
& -8 \pi T_{1}^{1}=\frac{1}{2} e^{-\beta-\gamma}\left[\frac{1}{2} \beta^{\prime 2}+\frac{2}{r^{2}}+\frac{2 \beta^{\prime}}{r}+\beta^{\prime} \alpha^{\prime}+\frac{2 \alpha^{\prime}}{r}\right] \\
& -e^{-\alpha}\left[\ddot{\beta}-\frac{1}{2} \dot{\beta} \dot{\alpha}+\frac{3}{4} \ddot{\beta}^{2}\right]-\frac{e^{-\beta}}{r^{2}}
\end{aligned}
$$

$$
\begin{aligned}
&-8 \pi T_{2}^{2}=-8 \pi T_{3}^{3}=\frac{1}{4} e^{-\beta-\gamma}\left[2 \alpha^{\prime \prime}+\alpha^{\prime 3}+2 \beta^{\prime \prime}+\frac{2 \beta^{\prime}}{r}+\frac{2 \alpha^{\prime}}{r}-\lambda^{\prime}\left(\beta^{\prime}+\alpha^{\prime}+\frac{2}{r}\right)\right] \\
&+\frac{1}{4} e^{-x}\left[2 \dot{\beta} \dot{\alpha}-3 \dot{\beta}^{2}-4 \ddot{\beta}+\dot{\lambda} \dot{\alpha}-3 \dot{\beta} \dot{\lambda}-2 \ddot{\lambda}-\ddot{\lambda}^{2}\right] \\
&-8 \pi T_{4}^{4}=e^{-\beta-\gamma}\left[\beta^{\prime \prime}+\frac{1}{4} \beta^{\prime 2}+\frac{2 \beta^{\prime}}{r}+\frac{1}{r^{2}}-\lambda^{\prime}\left(\frac{\beta^{\prime}}{r}+\frac{1}{r}\right)\right] \\
&-\frac{1}{2} e^{-\alpha}\left[\frac{3}{2} \dot{\beta}^{2}+\dot{\beta} \dot{\lambda}\right]-\frac{e^{-\beta}}{r^{2}} \\
&-8 \pi T_{4}^{1}=-8 \pi T_{1}^{4}=\frac{1}{4} e^{-\beta-\gamma}\left[-2 \dot{\beta}^{\prime}+\dot{\lambda}\left(\beta^{\prime}+\frac{2}{r}\right)+\dot{\alpha} \dot{\beta}\right]
\end{aligned}
$$

For a disruption of neutrality in aggregate of matter we take,

$T_{i}^{k}=M_{i}^{k}+E_{i}^{k}$

$M_{i}^{k}=(p+\rho) V_{i} V^{k}-p \delta_{i}^{k}$

$V^{1}=V^{2}=V^{3}=0, V_{i} V^{i}=1$

and $4 \pi E_{i}^{k}=-F^{k a} F_{i a}+\frac{1}{4} \delta_{i}^{k} F^{a b} F_{a b}$ with $F_{[i j ; k]}=0$ and $F_{; k}^{i k}=4 \pi J^{i}$

For spherically symmetric charge distribution we will have $F_{i k^{*}}$ Furthermore, since we are using comoving coordinates, the charge current vector $J^{i}$ will have components $\left(0,0,0, J^{4}\right)$. Then above equation it is required that;

$F_{; k}^{1 k}=4 \pi J^{1}=0$ with the form of $T_{i}^{k}$ given above, we find that. 


$$
\begin{aligned}
& T_{1}^{1}=-p-\frac{1}{8 \pi} F^{14} F_{14} T_{2}^{2}=T_{3}^{3}=-p+\frac{1}{8 \pi} F^{14} F_{14} T_{4}^{4} \\
& =\rho-\frac{1}{8 \pi} F^{14} F_{14}, T_{4}^{1}=T_{1}^{4}=0 .
\end{aligned}
$$

Thus, we derive the following equations:

$$
\left\{\begin{array}{c}
T_{4}^{1}=0 \\
T_{1}^{1}-T_{2}^{2}=\frac{1}{4 \pi} e^{-2 \beta} f^{2}(r) \\
T_{1}^{1}-T_{4}^{4}=-p-\rho
\end{array}\right.
$$

It shows that owing to quantization of space. Trigger it to demonstrate itself as physical properties of a particle state. Which depends on dependency on physical quantities of matter where $\rho$ is the macroscopic density of radiation at the point of interest, $p$ is the proper pressure. With $\rho=3 p$

Equation of state of matter under contraction of mass space is derived below. The undertaking uniform composition of matter and its opacity imply that $n$ and $\kappa$ are constant at the point of action of space. Thus,

$m=n^{2} M, l=\kappa n^{2} \mathrm{~L}, t=n R T$

Proportionality of $\kappa \propto n$ is self solicit.

Using the equation of state to eliminate the density and pressure, the differential equations become;

$$
\frac{d m}{d x}=\frac{4 \pi a}{3 R} \cdot \frac{\beta t^{3}}{1-\beta} \cdot x^{2}
$$

$$
\frac{d l}{d m}=\kappa \varepsilon
$$

Convective $\left\{\begin{array}{l}\frac{d t}{d x}=-\frac{G}{R} \cdot \frac{\beta(8-6 \beta)}{32-24 \beta-3 \beta^{2}} \cdot \frac{m}{x^{2}} \\ \frac{d \beta}{d x}=\frac{G}{R} \cdot \frac{3 \beta^{2}(1-\beta)}{32-24 \beta-3 \beta^{2}} \cdot \frac{m}{x^{2}}\end{array}\right.$

Radiative $\left\{\begin{array}{c}\frac{d \beta}{d x}=\frac{1}{4 \pi c R} \cdot \frac{\beta}{x^{2} t}\{l-4 \pi c G m(1-\beta)\} \\ \frac{d t}{d x}=-\frac{1}{16 \pi c R} \cdot \frac{\beta}{1-\beta} \cdot \frac{l}{x^{2}}\end{array}\right.$

Together with the expression for the energy released by contraction,

$$
\varepsilon=\frac{3 R T}{2 n} \frac{\partial\left(\ln T-8 y / 3-\frac{2}{3} \ln y\right)}{\partial r},
$$

Where, $y=\frac{1-\beta}{\beta}, \beta$ is the ratio of pressure of aggregated matter to total pressure, and $T$ is the time.

To these equations are added the boundary conditions $m=0, l=0$ at the center, and $t=0$ at the surface. The additional condition necessary at the surface has been taken as:

$$
\frac{\kappa L}{4 \pi c G M(1-\beta)}=1
$$

This is an approximation to the more precise condition;

$$
\left(\frac{T}{T_{e}}\right)^{4}=\frac{1}{2\left\{1-\frac{\kappa L}{4 \pi c G M(1-\beta)}\right\}}
$$

Which holds in a radiative region near the surface, in which $M$ and $L$ are taken as constant and in which $K P R^{2} / G M$ has the value $2 / 3$ when $T=T e$ (effective temperature). The composition (which enters only through $K$ and $p$ ) and the radius are removed from the energy Equations (47) and (50) in the following way. Defining quantity $K$ by:

$$
\frac{n^{2}}{\kappa K}=\frac{d}{d \tau}\left(\frac{1}{R}\right)
$$

Equation (50) is of the form;

$$
\varepsilon=\frac{3 R t}{2 \kappa K}
$$

The surface condition (51) shows that $\kappa L$ is constant during the contraction, that is,

$$
\frac{3 R M}{2 K} \int_{0}^{1} t d q=\text { const }
$$

It follows that $K$ is constant, and the contraction follows the usual pattern in which the reciprocal of the radius changes uniformly with time. Combination of (47) and (51) leads to the energy equation;

$$
\frac{d l}{d m}=\frac{3 R t}{2 K}
$$

Equations (46), (48), (49), and (52) are now independent of both radius and time, and so may be integrated to give a model in a state of consistent contraction.

\section{NEW RESULTS}

\section{Cause of zero-point energy}

Now by a combination of gravitational waves and the uncertainty principle we will derive the expression of the Casimir force. An uncertainty $\Delta m_{i}$ in $m_{i}$ produces an uncertainty $\Delta p$ in $p$, and therefore an uncertainty $\Delta m_{g}$ in $m_{g}$ is given by Equation (37) as:

$$
m_{g}=m_{i}-2\left[\sqrt{12\left(\frac{\Delta p}{m_{i} c}\right)^{2}}-1\right] m_{i}
$$

From the uncertainty principle for position and momentum, we know that the product of the uncertainties of the simultaneously measurable values of corresponding position and momentum components is at least of the order of magnitude of $\hbar$, i.e., $\Delta p \Delta r \sim \hbar$

Substituting the value of $\Delta p$ in Equation (37) we get.

$$
\hbar m_{g}=m_{i}-2\left[\sqrt{12\left(\frac{\hbar / \Delta m_{i} c^{2}}{m_{i} c}\right)^{2}}-1\right] m_{i}
$$

Therefore, if $\Delta r \ll \hbar / \Delta m_{i} c$ then the expression (53) reduces to: $\Delta m_{g} \cong-\frac{2 \hbar}{\Delta r r c}$ Note that $\Delta m_{g}$ does not depend on $m_{g}$. Consequently, the uncertainty $\Delta F$ 
in the gravitational force;

$F=-G m_{g} m_{g}^{\prime} / r^{2}$, will be given by

$\Delta F=-G \frac{\Delta m_{g} \Delta m^{\prime} g}{(\Delta r)^{2}}=-\left[\frac{2}{\pi(\Delta r)^{2}}\right] \frac{h c}{(\Delta r)^{2}}\left(G \frac{\hbar}{c^{3}}\right)$

The entity $\left(G \frac{\hbar}{c^{3}}\right)^{\frac{1}{2}}=1.61 \times 10^{-35} \mathrm{~m}$ is called the plank length, $l_{\text {planck }}$

(the length scale on which quantum fluctuations of the metric of the space-time are expected to be of order unity). Thus, we can write the

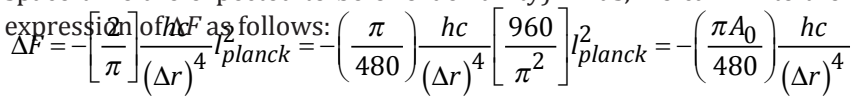

Or

$$
\Delta F=-\left(\frac{\pi A_{0}}{480}\right) \frac{h c}{(r)^{4}}
$$

Which is the expression of the Casimir force for $A=A_{0}=\left(\frac{960}{\pi^{2}}\right) l_{\text {planck }}^{2}$

This suggests that $A_{0}$ is an elementary area related to existence of a minimum length $d=n l_{\text {min planck }}$. Which is in accordance with the quantization of space which points out the existence of $d_{m i n}$. Now multiplying (55) by $n^{2}$ the expression of $F_{0}$ we obtain:

$$
F=n^{2} F_{0}=-\left(\frac{\pi n^{2} A_{0}}{480}\right) \frac{h c}{(r)^{4}}=-\left(\frac{\pi A}{480}\right) \frac{h c}{(r)^{4}}
$$

Which is the general expression of Casimir force. Thus, we conclude that the Casimir effect is just a gravitational effect related to the uncertainty principle.

\section{Expansion of universe}

The source of expansion of universe has always baffled physicist which they claim as the dark matter responsible for it. We have come up with quantize space. Now, the radiations emitted to space have the direct influence on the expansion of space itself. Let's see how?

The spotty Wilkinson microwave anisotropy probe data concluded the uniformity of cosmic microwave background radiation after the Big Bang. Let areas $A_{i}$ and $A_{j}$ area of space at quantized state. For the reason that of quantization of space it is supposed to demonstrate as physical properties of a particle state (already shown above). Thus, its scale for radius will vary of the order of the size of a particle. Thus, repulsive force $F_{\omega}$ produces is given as,

$$
F_{\omega}=\frac{A_{i} A_{j}}{4 \pi r^{2}} U
$$

Where, $U$ is the energy density of space. In terms of the temperature $T$,

$$
U=\frac{8 \pi h}{c^{3}} \int_{0}^{\infty} \frac{v^{3} d v}{\exp \left(\frac{h v}{k T}\right)-1}=\frac{8 \pi^{2} k^{4}}{15(h c)^{3}} T^{4}
$$

Which gives the Stefan-Boltzmann constant $\sigma$ in terms of the energy density of space,

$$
\sigma=\frac{c}{4 T^{4}} U=\frac{2 \pi^{2} k^{4}}{15 c^{2} h^{3}}
$$

The gravitational attraction force $F_{G}$ between the pair of masses,

$$
\begin{gathered}
F_{\text {repl }}=F_{\omega}-F_{G}=\frac{A_{i} A_{j}}{4 \pi r^{2}} U-\frac{m_{i} m_{j}}{R^{2}}=\frac{m_{i} m_{j}}{R^{2}}\left(\frac{U}{4 \pi} \frac{A_{i} A_{j}}{m_{i} m_{j}}-G\right) \\
\frac{A}{m}=\frac{A_{i}}{m_{i}}=\frac{A_{j}}{m_{j}}=\frac{3}{4 \rho r}
\end{gathered}
$$

Combining,

$$
F_{\text {repl }}=\frac{m_{i} m_{j}}{R^{2}}\left(\frac{U}{4 \pi r_{i} r_{j}}\left(\frac{3}{4 \rho}\right)^{2}-G\right)
$$

Hence, $F=F_{-}-F_{G}>0$. Radiated space is repulsive. However, the repulsion diminishes if the action of the point at space is larger on account of the presence of mass. For space filled with matter that we commonly observe on earth, the repulsion tends to vanish allowing gravitational attraction to dominate the net mass space pair interaction. Thus, the theory of dark matter rules out.

\section{Engineering gravitational waves}

Continuing our calculation from Equation (46). Replacing the equation of state by:

$\frac{\partial \lambda}{\partial t}=\ddot{\lambda}=0$ and $\alpha+\beta=0$

Then,

$E^{-\alpha}=e^{\beta}=(F+G)^{2}$

Where, $F=F(r)$ and $G=G(t)$

Thus, the field equation is:

$$
f(r)^{2}=\frac{1}{2} e^{\beta}\left[e^{-\lambda}\left\{\frac{1}{2} \beta^{\prime 2}-\frac{1}{2} \frac{\lambda^{\prime}}{r}-\frac{1}{r^{2}}\right\}+\frac{1}{r^{2}}\right]
$$

If $G=G(t)$ is not constant then, the above equation will resolve into:

$f(r)^{2}=F^{2} e^{-\lambda}$ and $e^{-\lambda}\left\{\frac{\lambda^{\prime}}{2 r}+\frac{1}{r^{2}}\right\}-\frac{1}{r^{2}}=0$

Where, $\mathrm{e}^{-\lambda}=1-k r^{2}, f(r)^{2}=F^{2}\left(1-k r^{2}\right)$

$k$ is an undetermined constant.

Hence, the modified solution of the field equation is given as:

$$
d s^{2}=(F+G)^{-2} d t^{2}-(F+G)^{2}\left[\frac{d r^{2}}{1-k r^{2}}+r^{2} d \Omega^{2}\right]
$$

Therefore

$$
8 \pi p=-\frac{k}{(F+G)^{2}}-2 \ddot{G}(F+G)-3 \ddot{G}^{2}
$$




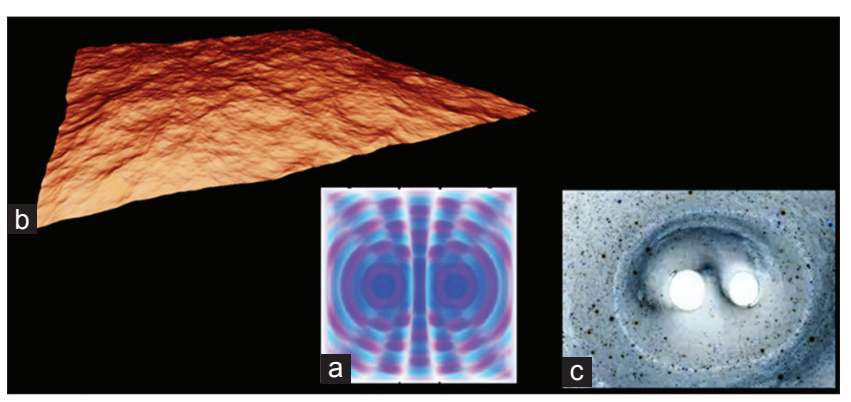

Fig. 1: Computational model of (a) two-dimensional and (b) threedimensional model of a (c) particle-driven gravitational waves current visualized by isosurfaces of concentration

$$
\begin{gathered}
8 \pi p=-2(F+G)^{-3}\left[F^{\prime \prime}\left(1-k r^{2}\right)+\left(2-3 k r^{2}\right) \frac{F^{\prime}}{r}\right]+3 k(F+G)^{-2}+3 \ddot{G}^{2} \\
F_{14}=\frac{F^{\prime}}{(F+G)^{2}}
\end{gathered}
$$

Consequently, $4 \pi \sigma=(F+G)^{-3}\left[F^{\prime \prime}\left(1-k r^{2}\right)+\left(2-3 k r^{2}\right) \frac{F^{\prime}}{r}\right]$ $\sigma$ is the charge density given by $\sigma=s\left|\left\{J^{a} J_{b}\right\}^{\frac{1}{2}}\right|$ (s is the sign of $\left.J^{4}\right)$.

Thus, we have established the formation of charges in a matter through the action of differed space mass quantization. Consequently, we know that dipole forces are dependent on the spacing between the two charges of the dipole and the orientation of the dipole with respect to the particle it acts on. What we all learned in introductory physics courses is that a balanced zero net charge particle is attracted to an external single charge such as an electron or a proton because the charges within the particle displace themselves to cancel the impinging field. The positively charged proton and the negatively charged electron in an atom cancel each other for all outside generation of electrostatic forces. This claim is incorrect due to a nonreciprocal characteristic of atoms. The magnitude and the sign (attraction or repulsion) of this nonzero force are determined by the relative resistivity and permittivity of the particle to the medium surrounding it. For example, a particle with either low resistivity or high permittivity relative to its background will be attracted to the dipole. Conversely, a particle with high resistivity and low permittivity relative to its background will be repulsed by the dipole. Hence, through our notion, there must be a nonzero force between dipole and the lone neutral particle. This differed space mass quantization creates a full-fledged stipulation to forge an astrophysical capacitor. Where the capacitance action of gravitational waves is experimentally demonstrated by late. Mr. Morton F. Spears. (Morton F. Spears, "An Electrostatic Solution for the Gravitational waves Force and the Value of G," January 9, 1997). Mathematically, we reached the goal to engineer gravitational waves in much complex paradigms and is validated in the LIGo experiment to simulate gravitational waves [18] (Fig. 1).

\section{CONCLUSION}

In the underlying subjects of interest, we presented an analysis of gravitation with "quantize field theory of gravitation" which drafts epistemological explanation of the origin of gravitational force through differed space mass quantization in consequent preservation of Einstein's geodesic equation and principle of equivalence. It provides an easy understanding of expansion of universe through the physical phenomena of radiative space extension and satisfactorily the cause of zero-point energy is revealed with uncertainty of gravitation at quantized scale. Subsequently, capacitance action of artificial gravitational waves with suited formulation is revealed which is already been experimentally justified.

\section{REFERENCES}

1. Spears MF. An Electrostatic Solution for the Gravitational waves Force and the Value of G. Galilean Electrodynamics; 1997.

2. Ungarish M. An Introduction to Gravitational Waves Currents and Intrusions. Boca Raton, FL: CRC Press; 2010.

3. Chassignet E, Cenedese C, Verron J. Buoyancy-Driven Flows. Cambridge, UK: Cambridge University Press; 2012.

4. Hopfinger EJ. Snow avalanche motion and related phenomena. Annu Rev Fluid Mech 1983;15(1):47-76.

5. Meiburg E, Kneller B. Turbidity currents and their deposits. Annu Rev Fluid Mech 2010;42:135-56.

6. Klaucke I, Hesse R, Ryan W. Seismic stratigraphy of the northwest atlantic mid-ocean channel: Growth pattern of a mid-ocean channellevee complex. Mar Pet Geol 1998;15(6):575-85.

7. Curray JR, Emmel FJ, Moore DG. The Bengal fan: Morphology, geometry, stratigraphy, history and processes. Mar Pet Geol 2002;19(10):1191-223

8. Weimer P, Slatt RM. Introduction to the Petroleum Geology of Deepwater Settings. Tulsa, OK: AAPG/Datapages; 2007.

9. Moncrieff MW. The dynamical structure of two-dimensional steady convection in constant vertical shear. Q J R Meteorol Soc 1978;104(441):543-67.

10. Maxworthy T, Leilich J, Simpson JE, Meiburg EH. The propagation of a gravitational waves current into a linearly stratified fluid. J Fluid Mech 2002;453:371-94.

11. Rottman J, Linden P. Gravitational waves currents. In: Grimshaw R, editor. Environmental Strati - Fied Flows. Dordrecht, Netherlands: Kluwer Academic; 2001. p. 89-117.

12. Rai A. Shell implementation of neural net over the UNIX environment for file management: A step towards automated operating system. J Oper Syst Dev Trends 2014;1(2):10-4.

13. Rai A. Automation of community from cloud computing. J Adv Shell Program 2014;1(1):21-3.

14. Rai A. Dynamic data flow based spatial sorting method for GPUs: Software based autonomous parallelization. Recent Trends Parall Comput 2014;1(1):15-8.

15. Rai A. Dynamic pagination for efficient memory management over distributed computational architecture for swarm robotics. J Adv Shell Program 2014;1(2):1-4.

16. Rai A, Ramanathan S, Kannan RJ. Quasi Opportunistic Supercomputing for Geospatial Socially Networked Mobile Devices. Enabling Technologies: Infrastructure for Collaborative Enterprises (WETICE), 2016 IEEE $25^{\text {th }}$ International Conference on. IEEE; 2016.

17. Baines P. Topographic Effects in Stratified Flows. Cambridge, UK: Cambridge University Press; 1995.

18. Available from: https://www.ligo.caltech.edu/video/ligo20160211v4. 